\title{
DESENVOLVIMENTO EM QUESTÃO
}

\section{Open Journal Systems}

\section{ISSN: 2237-6453}

\section{Assessores Cientificos/Avaliadores}

\section{(Relação atualizada em 10/2/2017: 432 doutores $(82 \%), 73$ doutorandos (14\%) e 23 mestres (4\%)}

- Dra. Adalmir de Oliveira Gomes, Universidade de Brasília, Brasil

- Dra. Adriana Carvalho Pinto Vieira, Universidade do Extremo Sul Catarinense, Brasil

- Dra. Adriana Maria de Aquino, Embrapa Agrobiologia, Brasil

- Dra. Adriana Marques Rossetto, Universidade Federal de Santa Catarina, Brasil

- Dra. Adriana Rochas de Carvalho Fruguli Moreira, Universidade Estadual de Mato Grosso do Sul, Brasil

- Dra. Adriana Roseli Wunsch Takahashi, Universidade Federal do Paraná, Brasil

- Dra. Adriana Soares Pereira, Universidade Federal de Santa Maria, Brasil

- Dr. Adriano Dias de Carvalho, Faculdade de Tecnologia, Mococa-SP, Brasil

- Dra. Aida Maria Lovison, Associação Nacional dos Filósofos Clínicos, Brasil

- Dr. Airton Adelar Mueller, Universidade Regional do Noroeste do estado do Rio Grande do Sul, Brasil

- Dr. Airton Cardoso Cançado, Universidade Federal do Tocantins, Brasil

- Dr. Alair Ferreira de Freitas, Universidade Federal de Viçosa, Brasil

- Doutorando Alberto Alves da Rocha, Instituto Nacional de Colonização e Reforma Agrária (Incra). Universidade Estadual de Ponta Grossa, PR, Brasil

- Doutoranda AlessanDra. Cassol, Universidade do Contestado, SC, Brasil 
- Doutoranda AlessanDra. Matte, Universidade Federal do Rio Grande do Sul, Brasil

- Dr. Aléssio Bessa Sarquis, Universidade do Sul de Santa Catarina, Brasil

- Dr. Alex AlexanDr.e Mengel, Universidade Federal do Rio Grande do Sul, Brasil

- Dr. Alex Pizzio, Universidade Federal do Tocantins, Brasil

- Dr. AlexanDr.e Cappellozza, Universidade Metodista de São Paulo, Brasil

- Dr. AlexanDr.e de Oliveira e Aguiar, Universidade Nove de Julho, SP, Brasil

- Dr. AlexanDr.e Godinho Bertoncello, Universidade do Oeste Paulista, Brasil

- Doutorando AlexanDr.e Hochmann Béhar, Universidade Federal de Pernambuco, Brasil

- Dr. AlexanDr.e Magno de Melo Faria, Universidade Federal de Mato Grosso, Cuiabá, Brasil

- Dr. AlexanDr.e Pereira Salgado Junior, Universidade de São Paulo, Ribeirão Preto, Brasil

- Dr. AlexanDr.e Rabelo Neto, Universidade Federal do Piauí, Brasil

- Msc Allan Carlos Alves, Universidade Estadual da Paraíba, Brasil

- Doutoranda Amábile Tolio Boessio, Universidade Federal de Santa Maria, Brasil

- Dra. Amazile López Netto, Universidade Federal Rural do Rio de Janeiro, Brasil

- Dra. Amarilis Lucia Casteli Figueiredo Gallardo, Universidade de São Paulo. Universidade Nove de Julho, SP, Brasil

- Doutoranda Ambrozina de Abreu Pereira Silva, Universidade Federal de Ouro Preto, Brasil

- Dra. Ana Claudia Machado Padilha, Universidade de Passo Fundo, Brasil

- Dra. Ana Cristina de Faria, Universidade Nove de Julho, SP, Brasil

- Doutoranda Ana Luisa Dal Belo Carneiro Leão, Universidade Federal de Sergipe, Brasil

- Dra. Ana Maria Bencciveni Franzoni, Universidade Federal de Santa Catarina, Brasil

- Dra. Ana Maria Colling, Universidade Federal da Grande Dourados, Brasil

- Dra. Ana Maria de Albuquerque Vasconcellos, Universidade da Amazônia, Brasil

- Dra. Ana Monteiro Costa, Universidade Federal de Pernambuco, Brasil

- $\quad$ Msc Ana Paula Alf Lima Ferreira, Universidade de Cruz Alta, Brasil 
- Dr. Ana Paula Lisboa Sohn, Universidade do Vale do Itajaí, SC, Brasil

- Dra. Ana Paula Paes de Paula, Universidade Federal de Minas Gerais, Brasil

- Dr. Anderson Felisberto Dias, Fundação Getúlio Vargas, Brasil

- $\quad$ Dr. Andre da Silva Pereira, Universidade de Passo Fundo, Brasil

- Dr. Andre Luís Assunção de Farias, Universidade Federal do Pará, Brasil

- Dr. Andre Luiz Marques Serrano, Universidade de Brasília, Brasil

- Dra. Andrea Eloisa Bueno Pimentel, Universidade Federal de São Carlos, Araras, Brasil

- Dra. Andréa Ferraz Fernandez, Universidade Federal do Mato Grosso, Brasil

- Doutoranda Andreia Carpes Dani, Universidade Regional de Blumenau, Brasil

- Doutoranda Andreia de Oliveira Santos, Centro Federal de Educação Tecnológica de Minas Gerais, Brasil

- Dra. Anete Alberton, Universidade do Vale do Itajaí, SC, Brasil

- Dra. Ângela Cristina Trevisan Felippi, Universidade de Santa Cruz do Sul, Brasil

- Dra. Angye Cássia Noia, Universidade Estadual de Santa Cruz, Ilhéus, Brasil

- Dra. Antônia Egídia de Souza, Universidade do Vale do Itajaí, SC, Brasil

- Msc. Antônio Augusto Brion Cardoso, Universidade Federal de Juiz de Fora, Brasil

- Dr. Antônio Carlos dos Santos, Universidade Federal de Lavras, Brasil

- Dr. Antonio Gil Costa Júnior, Universidade Federal do Rio Grande do Norte, Brasil

- Dr. Antonio Luiz Ribeiro Monteiro, Universidade de Pernambuco, Brasil

- Dr. Antonio Paulo Cargnin, Universidade Federal do Rio Grande do Sul. Secretaria do Planejamento, Mobilidade e Desenvolvimento Regional do Rio Grande do Sul, Brasil

- Dr. Argemiro Luís Brum, Universidade Regional do Noroeste do Estado do Rio Grande do Sul, Brasil

- Dra. Ariádne Scalfoni Rigo, Universidade Federal da Bahia, Brasil

- Dr. Arlindo Jesus Prestes de Lima, Universidade Federal de Santa Maria, Brasil

- Doutorando Arthur Breno Sturmer, Instituto Federal de Alagoas, Brasil

- Dr. Artur Gomes de Oliveira, Instituto Federal de Sergipe, Brasil 
- Dr. Augusto Fischer, Universidade do Oeste de Santa Catarina, Brasil

- Doutoranda Aurilene dos Santos Ferreira, Universidade Federal do Pará, Brasil

- Dra. Bárbara Françoise Cardoso, Universidade Estadual do Oeste do Paraná, Brasil

- Dr. Benedito Silva Neto, Universidade Federal da Fronteira Sul, Cerro Largo/ RS, Brasil

- Dr. Bruno César Melo Moreira, Instituto Federal de Minas Gerais, Formiga, Brasil

- Doutorando Bruno de Jesus Lopes, Universidade Federal de Viçosa, Brasil

- Dr. Caio César de Medeiros Costa, Universidade de Brasília, Brasil

- Doutoranda Carla Gabriela Cavini Bontempo, Embrapa Mandioca e Fruticultura. Universidade Federal de Santa Catarina, Brasil

- Dra. Carla Patrícia da Silva Souza, Centro Universitário Internacional Uninter, Brasil

- Dr. Carlos Aguedo Nagel Paiva, Fundação de Economia e Estatística Siegfried Emanuel Heuser, Porto Alegre. Faculdades Integradas de Taquara, RS, Brasil

- Dr. Carlos Alberto Marçal Gonzaga, Universidade Estadual do Centro Oeste, PR, Brasil

- Dr. Carlos Antonio Brandão, Universidade Federal do Rio de Janeiro, Brasil

- Dr. Carlos de Almeida Gonçalves, Universidade de Lisboa, Portugal

- Doutorando Carlos Frederico Kraemer, Universidade Federal Fluminense, Brasil

- Dr. Carlos Ricardo Rossetto, Universidade do Vale do Itajaí, SC, Brasil

- Dr. Carlos Roberto Sanquetta, Universidade Federal do Paraná, Brasil

- Dra. Carolina Andion, Universidade do Estado de Santa Catarina, Brasil

- Dra. Carolina Freddo Fleck, Universidade Federal do Pampa, Brasil

- Msc. Carolina Lindbergh Farias, Universidade Federal Rural de Pernambuco, Brasil

- Dra. Carolina Lopes Araújo, Universidade de Brasília, Brasil

- Doutoranda Caroline Mendonça Nogueira Paiva, Universidade Federal de Lavras, Brasil

- Dra. Cassiana Maris Lima Cruz, Universidade de Passo Fundo, Brasil 
- Dra. Celia Regina Orlandelli Carrer, Universidade de São Paulo, Pirassununga, Brasil

- $\quad$ Doutorando Celso Vanderlei, Universidade Nove de Julho, SP, Brasil

- Dr. César Augustus Winck, Universidade do Oeste de Santa Catarina, Brasil

- Dr. Cesar Eduardo Kroetz, Instituto Federal Farroupilha, RS, Brasil

- Dr. Cezar Augusto Miranda Guedes, Universidade Federal Rural do Rio de Janeiro, Brasil

- Dra. Cidonea Machado Deponti, Universidade de Santa Cruz do Sul, Brasil

- Dr. Christian Luiz da Silva, Universidade Tecnológica do Paraná, Brasil

- Dra. Christine da Silva Schröeder, Universidade Federal de Ciências da Saúde de Porto Alegre, Brasil

- Dra. Cláudia Echevenguá Teixeira, Uninversidade Nove de Julho, SP, Brasil

- Dra. Cláudia Fabiana Gohr, Universidade Federal da Paraíba, Brasil

- Dra. Cláudia Magalhães Dr.ouvot, Universidade de Salvador, Brasil

- Dra. Claudia Maria Prudêncio de Mera, Universidade de Cruz Alta, Brasil

- Dra. Cláudia Tirelli, Universidade de Santa Cruz do Sul, Brasil

- Dr. Claudio Reis Gonçalo, Universidade do Vale do Itajaí, Brasil

- Doutorando Claudio Vinicius Silva Farias, Instituto Federal do Rio Grande do Sul, Brasil

- Dr. Claudionor Guedes Laimer, Faculdade Meridional, Passo Fundo, Brasil

- Doutorando Cleiton Silva Ferreira Milagres, Universidade Federal do Tocantins, Brasil

- Dr. Clóvis Antônio Kronbauer, Universidade do Vale do Rio dos Sinos, Brasil

- Dr. Clóvis Ultramari, Pontifícia Universidade Católica do Paraná, Brasil

- Dra. Cristiane Froehlich, Universidade Feevale, RS, Brasil

- Dra. Cristina Dai Prá Martens, Universidade Nove de Julho, SP, Brasil

- $\quad$ Dra. Cristina de Souza Castro Lucas, Universidade de Brasília, Brasil

- Dra. Cristina Maria Dacach Fernandez Marchi, Universidade Católica do Salvador, Brasil

- Dra. Cyntia Meireles de Oliveira, Universidade Federal Rural da Amazônia, Brasil 
- Dra. Dalvanir Avelino Silva, Universidade Federal do Rio Grande do Norte, Brasil

- Doutoranda Damiana Machado de Almeida, Universidade Federal de Santa Maria, Brasil

- Dr. Daniel Arruda Coronel, Universidade Federal de Santa Maria, Brasil

- Dr. Daniel Calbino Pinheiro, Universidade Federal de São João del-Rei, Brasil

- Doutorando Daniel Claudy da Silveira, Universidade de Santa Cruz do Sul, Brasil

- Dr. Daniel Coelho Oliveira, Universidade Estadual de Montes Claros, MG, Brasil

- Doutorando Daniel de Cerqueira Lima e Penalva Santos, Instituto Federal de Pernambuco, Brasil

- Dr. Daniel Knebel Baggio, Universidade Regional do Noroeste do Estado do Rio Grande do Sul, Brasil

- Dr. Daniel Luciano Gevehr, Faculdades Integradas de Taquara, Brasil

- $\quad$ Msc. Daniel Penz, Universidade do Vale do Itajaí, Brasil

- Dr. Daniel RoDr.iguez de Carvalho Pinheiro, Universidade Estadual do Ceará, Brasil

- Dr. Daniel Rubens Cenci, Universidade Regional do Noroeste do Estado do Rio Grande do Sul, Brasil

- Dra. Daniela Dias Kühn, Universidade Federal de Santa Maria, Brasil

- Dra. Daniela Garcez Wives, Universidade Federal do Rio Grande do Sul, Brasil

- Dra. Danielle Regina Ullrich, Instituto Federal de Santa Catarina, Brasil

- Dra. Daniete Fernandes Rocha, Faculdade de Estudos Administrativos, MG, Brasil

- Dr. Dante Pinheiro Martinelli, Universidade de São Paulo, Brasil

- Dr. David Basso, Universidade Regional do Noroeste do Estado do Rio Grande do Sul, Brasil

- Dr. David Ferreira Lopes Santos, Universidade Estadual Paulista Júlio de Mesquita Filho, Brasil

- Doutoranda Dayane Clock, Instituto Federal de Santa Catarina, Brasil

- Dra. Déa de Lima Vidal, Universidade Estadual do Ceará, Brasil 
- Dra. Débora Coutinho Paschoal Dourado, Universidade Federal de Pernambuco, Brasil

- Dra. Débora Zumkeller Sabonaro, Universidade de Sorocaba, Brasil

- Dr. Dejalma Cremonese, Universidade Federal de Santa Maria, Brasil

- Dra. Luciene Eberle, Universidade de Caxias do Sul, Brasil

- Dra. Denise Barros de Azevedo, Universidade Federal de Mato Grosso do Sul, Brasil

- Dra. Denise Pereira Curi, Universidade Presbiteriana Mackenzie, Brasil

- Msc. Denise Rossato Quatrin, Faculdade de Direito de Santa Maria, Brasil

- Dra. Denize Grzybovski, Universidade de Passo Fundo, Brasil

- Dr. Dieter Rugard Siedenberg, Universidade Regional do Noroeste do Estado do Rio Grande do Sul, Brasil

- Dr. Dilamar Dallemole, Universidade Federal de Mato Grosso, Brasil

- Dr. Dilson Trennepohl, Universidade Regional do Noroeste do Estado do Rio Grande do Sul, Brasil

- Dr. Diogo Henrique Helal, Universidade Federal da Paraíba, Brasil

- Dr. Doglas Cesar Lucas, Universidade Regional do Noroeste do Estado do Rio Grande do Sul, Brasil

- Dra. Dolores Pereira Ribeiro Coutinho, Universidade Católica Dom Bosco, MS, Brasil

- Dr. Douglas Wegner, Universidade do Vale do Rio dos Sinos, Brasil

- Dra. Dunia Comerlatto, Universidade Comunitária da Região de Chapecó, Brasil

- Dr. Dusan Schreiber, Universidade Feevale, RS, Brasil

- Dr. Edegar Luis Tomazzoni, Universidade de São Paulo, Brasil

- Dr. Edgar Reyes Junior, Universidade de Brasília, Brasil

- Dr. Edson Arlindo Silva, Universidade Federal de Viçosa, Brasil

- Dr. Edson Keyso de Miranda Kubo, Universidade Municipal de São Caetano do Sul, Brasil

- Dr. Edson Ronaldo Guarido Filho, Universidade Positivo. Universidade Federal do Paraná, Brasil

- Dr. Edson Trajano Vieira, Universidade de Taubaté, SP, Brasil

- Dr. Edson Sadao Iizuka, Centro Universitário da FEI e Fecap, Brasil 
- Dr. Eduardo de Camargo Oliva, Universidade Municipal de São Caetano do Sul, Brasil

- Dr. Eduardo Gelinski Júnior, Universidade do Oeste de Santa Catarina, Brasil

- Dra. Elenise Felzke Schonardie, Universidade Regional do Noroeste do Estado do Rio Grande do Sul, Brasil

- Dra. Eliana AnDr.éa Severo, Faculdade Meridional, Passo Fundo, Brasil

- Dra. Eliane Salete Filippim, Universidade do Oeste de Santa Catarina, Brasil

- Dr. Elias Pereira Lopes Júnior, Universidade Federal do Cariri, Brasil

- Dra. Elisa Yoshie Ichikawa, Universidade Estadual de Maringá, Brasil

- Doutoranda Elisabeth Cristina Dr.umm, Universidade da Região da Campanha, RS, Brasil

- Dra. Elisete Dahmer Pfitscher, Universidade Federal de Santa Catarina, Brasil

- Dr. Elton Dias Xavier, Universidade Estadual de Montes Claros, MG, Brasil

- Doutorando Elvio Izaias da Silva, Universidade Federal de Santa Maria, Brasil

- Dr. Elzo Alves Aranha, Universidade Federal de Itajubá, MG, Brasil

- Dr. Emanuel Ferreira Leite, Universidade Católica de Pernambuco, Brasil

- Dr. Enio Waldir da Silva, Universidade Regional do Noroeste do Estado do Rio Grande do Sul, Brasil

- Dra. Enise Barth Teixeira, Universidade Federal da Fronteira Sul, Brasil

- Dr. Esteban Iglesias, Universidad Nacional de Rosario, Argentina

- Dra. Estela Najberg, Universidade Federal de Goiás, Brasil

- Dr. Ezequiel Redin, Universidade Federal de Santa Maria, Brasil

- Dr. Fabian Scholze Domingues, Universidade Federal do Rio Grande do Sul, Brasil

- Dra. Fabiana dos Santos Pereira Campos, Universidade Federal de Mato Grosso do Sul, Brasil

- Dra. Fabiana Marion Spengler, Universidade de Santa Cruz do Sul, Brasil

- Dr. Fabio Henrique Soares Angeoletto, Universidade Federal de Mato Grosso, Brasil

- Dr. Fábio Ytoshi Shibao, Universidade Nove de Julho, SP, Brasil

- Doutorando Fabrício Martins Lacerda, Universidade Nove de Julho, SP, Brasil 
- Dr. Fabricio Quadros Borges, Instituto Federal do Pará. Universidade da Amazônia, Brasil

- Dr. Fabricio Ziviani, Universidade Fumec, MG, Brasil

- Doutorando Felipe Ferreira de Lara, Universidade de São Paulo, Brasil

- Doutorando Felipe Fróes Couto, Universidade Estadual de Montes Claros, MG, Brasil

- Dra. Felisa Cançado Anaya, Universidade Estadual de Montes Claros, MG, Brasil

- Msc. Fernanda Cristina Sanches, Universidade Estadual do Oeste do Paraná, Brasil

- Dr. Fernando Antonio Aboim Freire Figueiredo, Instituto de Ensino Superior de Bauru, Brasil

- Dr. Fernando de Almeida Santos, Faculdades Metropolitanas Unidas. Pontifícia Universidade Católica de São Paulo, Brasil

- Dr. Fernando Guilherme Tenório, Fundação Getúlio Vargas, Brasil

- Msc. Fernando Vinícius da Rocha, Universidade de São Paulo, Brasil

- Dra. Flávia Luciana Naves Mafra, Universidade Federal de Lavras, Brasil

- Dr. Flávio Régio Brambilla, Universidade de Santa Cruz do Sul, Brasil

- Msc. Flavio Sérgio Rezende Nunes de Souza, Fundação Getúlio Vargas, Brasil

- Doutoranda Franciele Wrubel, Fundação Universidade Regional de Blumenau, Brasil

- Dr. Francisco Chagas Evangelista Rabêlo, Universidade Federal de Goiás, Brasil

- Doutorando Francisco de Tarso Ribeiro Caselli, Universidade Federal do Piauí, Brasil

- Dr. Francisco Giovanni David Vieira, Universidade Estadual de Maringá, Brasil

- Dr. Francisco Ricardo Duarte, Universidade Federal do Vale do São Francisco, Brasil

- Dr. Francisco Roberto Pinto, Universidade Estadual do Ceará, Brasil

- Dr. Frederico Fonseca da Silva, Instituto Federal do Paraná, Brasil

- Dr. Gabriel Sperandio Milan, Universidade de Caxias do Sul, Brasil

- Dra. Gabriela da Costa Silva, Universidade Federal do Rio de Janeiro, Brasil 
- Dra. Gabriela Gonçalves Silveira Fiates, Universidade Federal de Santa Catarina, Brasil

- Dr. Gelson Silva Junquilho, Universidade Federal do Espírito Santo, Brasil

- Dr. Geovanne Dias de Moura, Universidade Comunitária da Região de Chapecó, Brasil

- Dr. Gesinaldo Ataíde Cândido, Universidade Federal de Campina Grande, Brasil

- Dr. Geysler Rogis Flor Bertolini, Universidade Estadual do Oeste do Paraná, Brasil

- Doutoranda Giana de Vargas Mores, Universidade Federal do Rio Grande do Sul, Brasil

- Dr. Gilberto Ken Iti Yokomizo, Embrapa Amapá, Brasil

- Dr. Gilberto Perez, Universidade Presbiteriana Mackenzie, Brasil

- Dr. Gilmar Antonio Bedin, Universidade Regional do Noroeste do Estado do Rio Grande do Sul, Brasil

- Dr. Gilnei Luiz de Moura, Universidade Federal de Santa Maria, Brasil

- Doutoranda Giovana Goretti Feijó de Almeida, Universidade de Santa Cruz do Sul, Brasil

- Dra. Gisleia Benini Duarte, Universidade Federal Rural de Pernambuco, Brasil

- Doutoranda Glessia Silva, Universidade Federal de Alagoas, Brasil

- Dra. Grazielle Betina Brandt, Universidade de Santa Cruz do Sul, Brasil

- Doutorando Guélmer Junior Almeida Faria, Universidade Estadual de Montes Claros, Brasil

- Dr. Guillermo Javier Díaz-Villavicencio, Universidade Federal da Integração Latino-Americana, Brasil

- Dr. Gustavo da Rosa Borges, Universidade Federal do Pampa, RS, Brasil

- Msc Gustavo Henrique Silva de Souza, Instituto Federal do Norte de Minas Gerais, Brasil

- Doutorando Gustavo Leonardo Simão, Universidade Federal de Lavras, Brasil

- Dra. Heidy RoDr.iguez Ramos, Universidade Nove de Julho, SP, Brasil

- Dr. Hélio Zanquetto Filho, Universidade Federal do Espírito Santo, Brasil

- Doutoranda Heloiza Cristina Holgado da Silva, Universidade Estadual de Mato Grosso do Sul, Brasil 
- Msc. Henrique Bertosso, Faculdade de Administração da Associação Brasiliense de Educação, Brasil

- Dr. Heron Sergio Moreira Begnis, Universidade de Santa Cruz do Sul, Brasil

- Dr. Hubert Dr.ouvot, Université Pierre Mendès France, Grenoble, França

- Doutorando Hugo Alves Silva Ribeiro, Universidade de Brasília, Brasil

- Dr. Ignacio Tomás Trucco, Universidad Nacional del Litoral, Santa Fe, Argentina

- Msc. Igor Martins, Universidade Estadual da Paraíba, Brasil

- Msc. Iriane Teresa de Araújo, Universidade Federal Rural do Semi-Árido, Brasil

- Dra. Isa de Oliveira Rocha, Universidade do Estado de Santa Catarina, Brasil

- Dra. Isabel Cristina dos Santos, Universidade Municipal de São Caetano do Sul, Brasil

- Dra. Isabela Barchet, Universidade Estadual do Oeste do Paraná, Brasil

- Doutorando Itzhak David Simão Kaveski, Universidade Federal de Mato Grosso do Sul, Brasil

- Dr. Ivano Ribeiro, Universidade Estadual do Oeste do Paraná, Brasil

- Dr. Ivo Marcos Theis, Universidade Regional de Blumenau, Brasil

- Dr. Ivo Vasconcelos PeDr.osa, Universidade de Pernambuco, Brasil

- Dr. Jacir Favretto, Universidade do Contestado, Brasil

- Doutoranda Jaiane Aparecida Pereira, Universidade Estadual de Maringá, Brasil

- Doutoranda Jalva Lilia Rabelo Sousa, Instituto Federal do Piauí, Brasil

- Dra. Janaína Balk Brandão, Universidade Federal do Pampa, Brasil

- Doutoranda Janaina Ottonelli, Universidade Federal de Santa Maria, Brasil

- Dr. Jandir Ferrera de Lima, Universidade Estadual do Oeste do Paraná, Brasil

- Dr. Jandir Pauli, Faculdade Meridional, Passo Fundo, Brasil

- Dra. Janete Stoffel, Universidade Federal da Fronteira Sul Laranjeiras do Sul-PR, Brasil

- Doutorando Jesiel Souza Silva, Instituto Federal Goiano, Brasil

- Dra. Joana Tereza Vaz de Moura, Universidade Federal do Rio Grande do Norte, Brasil

- Dr. João AlexanDr.e Paschoalin Filho, Universidade Nove de Julho, SP, Brasil 
- Dr. João Batista Pamplona, Universidade Municipal de São Caetano do Sul. Pontifícia Universidade Católica de São Paulo, Brasil

- Doutorando João Carlos Hipólito Bernardes do Nascimento, Universidade Federal do Rio de Janeiro, Brasil

- Dr. João Carlos Tedesco, Universidade de Passo Fundo, Brasil

- Dr. João Eduardo Prudêncio Tinoco, Faculdade Campo Limpo Paulista, Brasil

- Dr. João Mendes Rocha Neto, Universidade de Brasília, Brasil

- Dr. Joel Orlando Bevilaqua Marin, Universidade Federal de Santa Maria, Brasil

- Dr. Jorge Luiz Amaral de Moraes, Universidade de Santa Cruz do Sul. Faculdades Integradas de Taquara, Brasil

- Dr. Jorge Oneide Sausen, Universidade Regional do Noroeste do Estado do Rio Grande do Sul, Brasil

- Dr. Jose Alberto Carvalho dos Santos Claro, Universidade Federal de São Paulo, Brasil

- Dr. José AnDr.é Villas Boas Mello, Centro Federal de Educação Tecnológica Celso Zuckow da Fonseca, RJ, Brasil

- Dr. Jose Carlos Lazaro da Silva Filho, Universidade Federal do Ceará, Brasil

- Dr. José Carlos Martines Belieiro Junior, Universidade Federal de Santa Maria, Brasil

- Dr. Jose Carlos Thomaz, Universidade Presbiteriana Mackenzie, SP, Brasil

- Dr. José Carlos Vaz, Universidade de São Paulo, Brasil

- Dr. Jose Elmar Feger, Universidade Federal do Paraná, Brasil

- Dr. José Irivaldo Alves Oliveira Silva, Universidade Federal de Campina Grande, Brasil

- Dr. José PeDr.o Cabrera Cabral, Universidade Federal do Tocantins, Brasil

- Dr. José Roberto Pereira, Universidade Federal de Lavras, Brasil

- Dr. Josiel Valadares, Universidade Federal de Juiz de Fora, Brasil

- Dr. Josmar Gilberto Cappa, Pontifícia Universidade Católica de Campinas, Brasil

- Dra. Josmária Lima Ribeiro de Oliveira, Pontifícia Universidade Católica de Minas Gerais, Brasil

- Dr. Juan Vicente Bachiller, Universidade Federal Fluminense, Brasil. FLACSO, Espanha 
- Doutoranda Juliana Gonçalves de Araújo, Universidade Federal de Pernambuco Brasil

- Dra. Juliana Magalhães Christino, Faculdade de Estudos Administrativos, Brasil

- Doutorando Juscelino Gomes Lima, Universidade de Santa Cruz do Sul, Brasil

- Dra. Jussara Maria Silva, Universidade Positivo, Brasil

- Dra. Karen Beltrame Becker Fritz, Universidade de Passo Fundo, Brasil

- Dra. Kellen da Silva Coelho, Universidade Federal da Paraíba, Brasil

- Dra. Kelmara Mendes Vieira, Universidade Federal de Santa Maria, Brasil

- Dr. Kenny Basso, Faculdade Meridional, Passo Fundo, Brasil

- Msc. Kenyth Alves de Freitas, Universidade Federal de Minas Gerais, Brasil

- Doutoranda Laila Mayara Dr.ebes, Universidade Federal de Santa Maria, Brasil

- Dra. Laís Karla da Silva Barreto, Universidade Potiguar, Brasil

- Dr. Lamartine Vieira Braga, Fundação Getúlio Vargas, Brasil

- Dr. Lamounier Erthal Villela, Universidade Federal Rural do Rio de Janeiro, Brasil

- Dra. Larissa Bueno Ambrosini, Fundação Estadual de Pesquisa Agropecuária, RS, Brasil

- Dr. Lauro Nalini, Pontifícia Universidade Católica de Goiás, Brasil

- Dr. LeanDr.o Augusto Toigo, Fundação Universidade Regional de Blumenau, Brasil

- Dr. LeanDr.o Petarnella, Universidade Nove de Julho, SP, Brasil

- Dra. Leci Martins Menezes Reis, Instituto Federal do Rio Grande do Norte, Brasil

- Dr. Leonardo Francisco Figueiredo Neto, Universidade Federal de Mato Grosso do Sul, Brasil

- Dr. Leonardo Xavier da Silva, Universidade Federal do Rio Grande do Sul, Brasil

- Dr. Leonel Mazzali, Centro Universitário das Faculdades Metropolitanas Unidas, SP, Brasil

- Dra. Lígia Maria Heinzmann, Universidade Federal de Mato Grosso, Brasil

- Dra. Lilian Lopes Ribeiro, Universidade Federal do Ceará, Brasil

- Dra. Liliane Cristine Schlemer Alcântara, Universidade Regional de Blumenau, Brasil 
- Dra. Liliane Oliveira Guimarães, Pontifícia Universidade Católica de Minas Gerais, Brasil

- Dr. Lucas Bonacina Roldan, Pontifícia Universidade Católica do Rio Grande do Sul, Brasil

- Dr. Lucas Labigalini Fuini, Universidade Estadual Paulista, Ourinhos, Brasil

- Dra. Lucélia Ivonete Juliani, Universidade Regional Integrada do Alto Uruguai e das Missões, Brasil

- Dra. Lúcia Kratz, Faculdade Alves Faria, Brasil

- Dra. Lúcia Rejane da Rosa Gama MaDr.uga, Universidade Federal de Santa Maria, Brasil

- Doutoranda Luciana Aparecida Barbieri da Rosa, Universidade Federal de Santa Maria, Brasil

- Dra. Luciana Flores Battistella, Universidade Federal de Santa Maria, Brasil

- Dr. Luciano Mendes, Universidade de São Paulo, Esalq, Brasil

- Dr. Luciano Venelli-Costa, Universidade Metodista de São Paulo, Brasil

- Dr. Lucir Reinaldo Alves, Universidade do Oeste do Paraná, Brasil

- Dr. Luis Carlos Zucatto, Universidade Federal de Santa Maria, Palmeira das Missões, Brasil

- Dr. Luís Moretto Neto, Universidade Federal de Santa Catarina, Brasil

- Dr. Luís Otávio Bau Macedo, Universidade Federal de Mato Grosso, Rondonópolis, Brasil

- Dr. Luis Paulo Bresciani, Universidade Municipal de São Caetano do Sul, Brasil

- Dr. Luis Roque Klering, Universidade Federal do Rio Grande do Sul, Brasil

- Dr. Luiz Alberto LeanDr.o, Universidade Federal Rural do Rio de Janeiro, Brasil

- Dr. Luiz Alex Silva Saraiva, Universidade Federal de Minas Gerais, Brasil

- Dr. Luiz AlexanDr.e Gonçalves Cunha, Universidade Estadual de Ponta Grossa-PR, Brasil

- Dr. Luiz Antônio Abrantes, Universidade Federal de Viçosa, Brasil

- Dr. Luiz Antonio Mafra, Universidade Federal de Alfenas, Varginha, MG, Brasil

- Dr. Luiz Fernando Fritz Filho, Universidade de Passo Fundo, Brasil

- Dr. Luiz Honorato da Silva Júnior, Universidade de Brasília, Brasil 
- Dra. Lurdes Marlene Seide Froemming, Universidade Regional do Noroeste do Estado do Rio Grande do Sul, Brasil

- Dr. Magnus Emmendoerfer, Universidade Federal de Viçosa, Brasil

- Msc. Mara Cristine Kich, Faculdade Borges de Mendonça e Unisul, Brasil

- Doutorando Marcelo de Moraes Cordeiro, Pontifícia Universidade Católica do Rio Grande do Sul, Brasil

- Dr. Marcelo Luiz Gabriel, Universidade Nove de Julho, SP, Brasil

- Dr. Marcelo Santana Silva, Instituto Federal da Bahia, Santo Amaro, Brasil

- Dra. Márcia da Silva Costa, Universidade Federal da Paraíba, Brasil

- Dra. Márcia Freire de Oliveira, Universidade Federal de Uberlândia, Brasil

- Doutoranda Marcia Helena dos Santos Bento, Universidade Federal de Santa Maria, Brasil

- Dra. Márcia Luíza Pit Dal Magro, Universidade Comunitária da Região de Chapecó, Brasil

- Dra. Márcia Zampieri Grohmann, Universidade Federal de Santa Maria, Brasil

- Dra. Marcilene Feitosa Araújo, Universidade Federal do Sul e Sudeste do Pará, Brasil

- Dr. Márcio de Araújo Pereira, Universidade Estadual de Mato Grosso do Sul, Brasil

- Dr. Marco AnDr.é Cadona, Universidade de Santa Cruz do Sul, Brasil

- Dr. Marco Antônio Carvalho Teixeira, Fundação Getúlio Vargas, Brasil

- Dr. Marco Antônio Verardi Fialho, Universidade Federal de Santa Maria, Brasil

- Dr. Marco Aurélio Batista de Sousa, Universidade Federal de Mato Grosso do Sul, Brasil

- Msc. Marco Aurélio da Silva, Faculdade do Baixo Parnaíba, MA, Brasil

- Dr. Marco Aurélio Marques Ferreira, Universidade Federal de Viçosa, Brasil

- Dr. Marco Tulio Aniceto Franca, Pontifícia Universidade Católica do Rio Grande do Sul, Brasil

- Dr. Marcos AlexanDr.e Luciano, Universidade de Caxias do Sul, Brasil

- Dr. Marcos Alves Magalhães, Centro Universitário de Caratinga, MG, Brasil

- Doutorando Marcos Antônio Souza dos Santos, Universidade Federal Rural da Amazônia, Brasil 
- Dr. Marcos Barcellos de Souza, Universidade Federal do ABC, SP, Brasil

- Msc. Marcos Júnior de Moura Paula, Banco do Brasil S.A., Brasil

- Dr. Marcos Junior Marini, Universidade Tecnológica Federal do Paraná, Brasil

- Dr. Marcos Paulo Dhein Griebeler, Faculdades Integradas de Taquara, Brasil

- Dr. Marcus Vinicius de Oliveira Brasil, Universidade Federal do Cariri, Brasil

- Dr. Marcus Vinicius Moreira Zittei, Centro Universitário Fecap, Brasil

- Dra. Maria Cristina Sanches Amorim, Pontifícia Universidade Católica de São Paulo, Brasil

- Dra. Maria de Lourdes Bernartt, Universidade Tecnológica Federal do Paraná, Brasil

- Dra. Maria do Carmo Meirelles Toledo Cruz, Fundação Getúlio Vargas, Brasil

- Doutoranda Maria Eloisa Karolczak, Universidade do Estado de Mato Grosso, Brasil

- Dra. Maria Elza de AnDra.de, Universidade do Estado do Rio Grande do Norte, Brasil

- Dra. Maria Emília Camargo, Universidade de Caxias do Sul, Brasil

- Dra. Maria Fernanda Gatto, Universidade Federal de Pernambuco, Brasil

- Dra. Maria Gracinda Carvalho Teixeira, Universidade Federal Rural do Rio de Janeiro, Brasil

- Doctoranda María Laura Freyre, Universidad Nacional de Córdoba, Argentina

- Dra. Maria Ligia Cassol Pinto, Universidade Estadual de Ponta Grossa, Brasil

- Dra. Maria Luiza Milani, Universidade do Contestado, SC, Brasil

- Dra. Maria Rita Loureiro, Fundação Getúlio Vargas de São Paulo, Brasil

- Dra. Maria Teresa Bustamante, Universidade Sociedade Educacional de Santa Catarina, Brasil

- Dra. Maria Valentina Locher, Universidad Nacional del Litoral, Argentina

- Dr. Mariano Yoshitake, Faculdades Alves Faria, Brasil

- $\quad$ Dra. Mariluce Paes-de-Souza, Universidade Federal de Rondônia, Brasil

- Dr. Mário Vasconcellos Sobrinho, Universidade Federal do Pará. Universidade da Amazônia, Brasil

- Dra. Mariza Costa Almeida, Universidade Federal do Estado do Rio de Janeiro, Brasil 
- Dra. Marta Elisete Ventura da Motta, Universidade de Caxias do Sul, Brasil

- Dra. Martha A. Saad Lucchesi, Universidade de São Paulo, Brasil

- Dr. Martinho Luís Kelm, Universidade Regional do Noroeste do Estado do Rio Grande do Sul, Brasil

- Dra. Mary SanDra. Guerra Ashton, Universidade Feevale, RS, Brasil

- Msc. Matheus Frohlich Marquetto, Universidade Federal de Santa Maria, Brasil

- Dr. Mauro Silva Ruiz, Universidade Nove de Julho, SP, Brasil

- Dra. Melissa Franchini Cavalcanti-Bandos, Centro Universitário Municipal de Franca, Brasil

- Dra. Melody de Campos Soares Porsse, Universidade Federal do Paraná, Brasil

- Dr. Miguel Angel Verdinelli, Universidade do Vale do Itajaí, Brasil

- Dr. Miguel Rivera Peres Júnior, Instituto Federal de Minas Gerais, Formiga, Brasil

- Dr. Milton Cordeiro Farias Filho, Universidade da Amazônia, Brasil

- Dr. Milton Luiz Wittmann, Universidade de Santa Cruz do Sul, Brasil

- Dr. Minelle Enéas da Silva, Universidade de Fortaleza, Brasil

- Dra. Mirelle Cristina de Abreu Quintela, Universidade Federal dos Vales do Jequitinhonha e Mucuri, Brasil

- Dra. Mirian Beatriz Schneider, Universidade Estadual do Oeste do Paraná, Brasil

- Dr. Moacir Francisco Deimling, Universidade Federal da Fronteira Sul, Brasil

- Dr. Moacir José dos Santos, Universidade de Taubaté, SP, Brasil

- Dr. Moacir Piffer, Universidade Estadual do Oeste do Paraná, Brasil

- Dra. Moema Pereira Nunes, Pontifícia Universidade Católica do Rio Grande do Sul, Brasil

- Dra. Mônica Aparecida da Rocha Silva, Universidade Federal do Tocantins, Brasil

- Dra. Mônica Cavalcanti Sá de Abreu, Universidade Federal do Ceará, Brasil

- Dra. Monica Franchi Carniello, Universidade de Taubaté, SP, Brasil

- Doutoranda Mônica Luize Sarabia, Universidade Federal de Pernambuco, Brasil

- Dra. Mônica Mota Tassigny, Universidade de Fortaleza, Brasil

- Dra. Naisy Silva Soares, Universidade Estadual de Santa Cruz, Ilhéus-BA, Brasil 
- Dra. Nathalia Carvalho Moreira, Universidade Federal de Sergipe, Brasil

- Doutoranda Nayara Pasqualotto, Universidade Federal de Santa Maria, Brasil

- Dra. Neli Galarce Machado, Centro Universitário Univates, Brasil

- Dr. Nelson Giordano Delgado, Universidade Federal Rural do Rio de Janeiro, Brasil

- Dr. Nelson Jose Thesing, Universidade Regional do Noroeste do Estado do Rio Grande do Sul, Brasil

- Dra. Neuza Abbud Prado Garcia, Universidade Nove de Julho, SP, Brasil

- Dr. Nilson Luiz Costa, Universidade Federal de Santa Maria, Brasil

- Dr. Nilton Cesar Lima, Universidade Federal de Uberlândia, Brasil

- Dr. Nilton Marques Oliveira, Universidade Federal do Tocantins, Brasil

- $\quad$ Dra. Noëlle Marie Paule Lechat, pesquisadora aposentada, Natal/RN, Brasil

- Dr. Nonato Assis de Miranda, Universidade Municipal de São Caetano do Sul, Brasil

- Dr. Norberto Martins Vieira, Universidade Federal de São João del-Rei, Brasil

- Dr. Olivier François Vilpoux, Universidade Católica Dom Bosco, Campo Grande, MS, Brasil

- Dr. Osmar Manoel Nunes, Universidade Federal do Pampa, Brasil

- Dr. Pascoal José Marion Filho, Universidade Federal de Santa Maria, Brasil

- Doutoranda Paula Patrícia Ganzer, Universidade de Caxias do Sul, Brasil

- Dr. Paulo Ricardo Feistel, Universidade Federal de Santa Maria, Brasil

- Dr. Paulo Roberto Cecconi Deon, Instituto Federal Farroupilha São Vicente do Sul, Brasil

- Dr. Paulo Sergio Gonçalves de Oliveira, Universidade Ibirapuera, Brasil

- Dr. PeDr.o Luís Büttenbender, Universidade Regional do Noroeste do Estado do Rio Grande do Sul, Brasil

- Dr. PeDr.o Selvino Neumann, Universidade Federal de Santa Maria, Brasil

- Dra. Priscila Cembranel, Universidade do Vale do Itajaí, Brasil

- $\quad$ Dra. Priscila Rezende da Costa, Universidade Nove de Julho, SP, Brasil

- Doutoranda Rafaella Stradiotto Vignandi, Universidade Federal de Minas Gerais, Brasil 
- Dr. Raimundo Eduardo Silveira Fontenele, Universidade de Fortaleza. Universidade Federal do Ceará, Brasil

- Dra. Raquel Breitenbach, Instituto Federal do Rio Grande do Sul, Sertão, Brasil

- Doutoranda Raquel Kohler, Universidade Federal de Sergipe, Brasil

- Dra. Raquel da Silva Pereira, Universidade Municipal de São Caetano do Sul, Brasil

- Dr. Reisoli Bender Filho, Universidade Federal de Santa Maria, Brasil

- Doutorando Renan Felinto de Farias Aires, Universidade Federal do Rio Grande do Norte, Brasil

- $\quad$ Doutorando Renato Fabiano Cintra, Universidade Nove de Julho, SP, Brasil

- Dr. Renato Linhares de Assis, Centro Nacional de Pesquisa de Agrobiologia, Embrapa, Brasil

- Dr. Ricardo Massulo Albertin, Universidade de Engenharia e Inovação Técnico Profissional, Brasil

- Dr. Richard Medeiros Araújo, Centro Universitário Facex, RN, Brasil

- Dr. Richardson Coimbra Borges, Centro Universitário de Lavras, Brasil

- Dra. Rita Ines Paetzhold Pauli, Universidade Federal de Santa Maria, Brasil

- Dr. Roberto Carlos Klann, Universidade Regional de Blumenau, Brasil

- Dr. Roberto de Gregori, Universidade Federal de Santa Maria, Brasil

- Dr. Roberto Giro Moori, Universidade Presbiteriana Mackenzie, SP, Brasil

- Dr. Robson Antônio Tavares Costa, Universidade Federal do Amapá, Brasil

- Msc Robson Fernandes Barbosa, Universidade Federal de Campina Grande, Brasil

- Dr. Rodolfo Coelho Prates, Universidade Positivo, Curitiba/PR, Brasil

- Dr. Rodrigo Manoel Dias da Silva, Universidade Federal da Fronteira Sul, Brasil

- Doutorando Roger Augusto Luna, Fundação Getúlio Vargas, Brasil

- Dr. Rogério Allon Duenhas, Universidade Tecnológica Federal do Paraná, Brasil

- Dr. Rogério Eduardo Rodrigues Bazi, Pontifícia Universidade Católica de Campinas, Brasil

- Dr. Rogério Leandro Lima da Silveira, Universidade de Santa Cruz do Sul, Brasil

- Dr. Rogério Santos da Costa, Universidade do Sul de Santa Catarina, Brasil 
- Dr. Rógis Juarez Bernardy, Universidade do Oeste de Santa Catarina, Brasil

- Dr. Romualdo Kohler, Universidade Regional do Noroeste do Estado do Rio Grande do Sul, Brasil

- Dr. Ronaldo Guedes de Lima, Instituto Federal do Paraná, Campo Largo, Brasil

- Dr. Ronaldo Raemy Rangel, Fundação Getúlio Vargas, Brasil

- Dra. Rosana da Rosa Portella Tondolo, Universidade Federal de Pelotas, Brasil

- Dra. Rosane Bernardete Brochier Kist, Universidade de Santa Cruz do Sul., Brasil

- Dra. Rosani Marisa Spanevello, Universidade Federal de Santa Maria, Brasil

- Msc. Rosiane Oswald Flach, Faculdades de Itapiranga, Brasil

- Dra. Rosinha da Silva Machado Carrion, Universidade Federal do Rio Grande do Sul, Brasil

- Dra. Rossana Guerra Souza, Universidade Federal da Paraíba, Brasil

- Doutoranda Rossandra Oliveira de Bitencourt, Universidade Federal do Paraná, Brasil

- Msc. Rozali Araujo dos Santos, Universidade de Cruz Alta, Brasil

- Doutorando Sadraque Oliveira Rios, Universidade Federal da Bahia, Brasil

- Dr. Sandino Hoff, Universidade Anhanguera Uniderp, Brasil

- Dra. Sandra Beatriz Vicenci Fernandes, Universidade Regional do Noroeste do Estado do Rio Grande do Sul, Brasil

- Msc. Sandra Díaz Arreguín, Sabes, México

- Dra. Sandra. Mara Schiavi Bankuti, Universidade Estadual de Maringá, Brasil

- Dra. Sandra. Maria Santos, Universidade Federal do Ceará, Brasil

- Dr. Sandro Luiz Bazzanella, Universidade do Contestado, Brasil

- Doutoranda Sarah Farias Andrade, Universidade Estadual de Santa Cruz, BA, Brasil

- Dra. Selene Herculano, Universidade Federal Fluminense, Brasil

- Msc. Sérgio Adriany Santos Moreira, Instituto Federal do Espírito Santo, Brasil

- Dr. Sérgio Castro Gomes, Universidade da Amazônia, Brasil

- Dr. Sergio Duarte de Castro, Pontifícia Universidade Católica de Goiás, Brasil 
- Dr. Sérgio Luís Allebrandt, Universidade Regional do Noroeste do Estado do Rio Grande do Sul, Brasil

- Dr. Sérgio Luiz do Amaral Moretti, Universidade Anhembi Morumbi, Brasil

- Doutoranda Sheila Patrícia Ramos Beckhauser, Fundação Universidade Regional de Blumenau, Brasil

- Doutoranda Silvana Kloster, Universidade Estadual de Ponta Grossa, Brasil

- Dr. Silvio Antônio Ferraz Cário, Universidade Federal de Santa Catarina, Brasil

- Dra. Simone Aquino, Universidade Nove de Julho, SP, Brasil

- Dra. Simone Bochi Dorneles, Instituto Federal Farroupilha, São Vicente do Sul, Brasil

- Dra. Simone Sehnem, Universidade do Oeste de Santa Catarina. Universidade do Sul de Santa Catarina, Brasil

- Dra. Sirlei Favero Cetolin, Universidade do Oeste de Santa Catarina, Brasil

- Dra. Sirlei Tereza Pitteri Vieira, Universidade de São Paulo, Brasil

- Dra. Sirlei Tonello Tisott, Universidade Federal de Mato Grosso do Sul, Brasil

- Dra. Solange Regina Marin, Universidade Federal de Santa Maria, Brasil

- Dra. Sonia Valle Walter Borges de Oliveira, Universidade de São Paulo, Ribeirão Preto, Brasil

- Dra. Stela Luiza de Mattos Ansanelli, Universidade Estadual Paulista Júlio de Mesquita Filho, Araraquara, Brasil

- Dra. Suely de Fátima Ramos Silveira, Universidade Federal de Viçosa, Brasil

- Dra. Suzete Antonieta Lizote, Universidade do Vale do Itajaí, Brasil

- Dra. Taisa Dias, Universidade do Sul de Santa Catarina, Brasil

- Dr. Tales Wanderley Vital, Universidade Federal Rural de Pernambuco, Brasil

- Dra. Tamara Cecilia Karawejczyk, Centro Universitário La Salle, Canoas, Brasil

- Dra. Tamara Silvana Menuzzi Diverio, Universidade de Cruz Alta, Brasil

- Dra. Tânia Maria de Oliveira Almeida Gouveia, Universidade Estadual do Rio de Janeiro, Brasil

- Dra. Tania Nunes Silva, Universidade Federal do Rio Grande do Sul, Brasil

- Dra. Tania Regina Frota Vasconcellos Dias, Universidade Federal Rural do Rio de Janeiro, Brasil

- Dra. Tanice Andreatta, Universidade Federal de Santa Maria, Brasil 
- Dra. Tanise Dias Freitas, Universidade Federal do Rio Grande do Sul, Brasil

- $\quad$ Dra. Tatiana Ghedine, Universidade do Vale do Itajaí, Brasil

- Doutoranda Tatiani Sobrinho Del Bianco, Universidade Estadual do Oeste do Paraná, Brasil

- Dr. Telmo Rudi Frantz, Universidade Regional do Noroeste do Estado do Rio Grande do Sul, Brasil

- Dra. Temis Gomes Parente, Universidade Federal do Tocantins, Brasil

- Dra. Thaís Ettinger Salgado, Centro Universitário Senac, Brasil

- Dra. Thais Soares Kronemberger, Universidade Federal Fluminense, Volta Redonda, Brasil

- Dra. Thelma Pontes Borges, Universidade Federal do Tocantins, Brasil

- Doutoranda Thalita Silva Calíope, Universidade Federal do Ceará, Brasil

- Dr. Thiago Alves, Universidade Federal de Goiás, Brasil

- Dr. Thiago Duarte Pimentel, Universidade Federal de Juiz de Fora, MG

- Dr. Thiago Ferreira Dias, Universidade Federal do Rio Grande do Norte, Brasil

- Dr. Thiago Fontelas Rosado Gambi, Universidade Federal de Alfenas, MG, Brasil

- Dr. Thiago Periard do Amaral, Universidade Federal de São João del-Rei, Brasil

- Dr. Thiago Soares Nunes, Universidade Federal de Santa Catarina, Brasil

- Dr. Thomas Ludewigs, Universidade de Brasília, Brasil

- Dr. Tiago Savi Mondo, Instituto Federal de Santa Catarina, Brasil

- Doutorando Tomás Amaral Torezani, Fundação de Economia e Estatística, RS, Brasil

- Dr. Valdenildo Pedro da Silva, Instituto Federal do Rio Grande do Norte, Brasil

- Doutorando Valderí de Castro Alcântara, Universidade Federal de Lavras, Brasil

- Dr. Valdir Frigo Denardin, Universidade Federal do Paraná, Brasil

- Dr. Valdir Roque Dallabrida, Universidade do Contestado, SC, Brasil

- Doutoranda Valéria da Veiga Dias, Universidade Federal do Rio Grande do Sul, Brasil

- Doutoranda Vanessa Theis, Universidade Feevale, Brasil

- Dra. Vânia Lúcia Quadros Nascimento, Universidade Federal do Pará, Brasil 
- Doutoranda Vanilda Barbosa Galli, Universidade da Região de Joinville, Brasil

- Doutoranda Veronica Eberle, Universidade Positivo, Curitiba, Brasil

- Dra. Verônica Macário de Oliveira, Universidade Federal de Campina Grande, Brasil

- Dr. Vicente Celestino Pires Silveira, Universidade Federal de Santa Maria, Brasil

- Dr. Victor Ramiro Fernández, Universidad Nacional del Litoral, Santa Fe, Argentina

- Dr. Victor Silva Corrêa, Pontifícia Universidade Católica de Minas Gerais, Brasil

- Dr. Vilmar Antonio Gonçalves Tondolo, Fundação Universidade Federal do Rio Grande, Brasil

- Dr. Vitor Koki da Costa Nogami, Fundação Instituto de Administração, Brasil

- Dra. Viviane Guimarães Pereira, Universidade Federal de Itajubá, MG, Brasil

- Doutoranda Vivianne Pereira Salas Roldan, Centro Universitário Estácio do Ceará, Brasil

- Dra. Viviane Rossato Laimer, Anhanguera Educacional e Faculdade Ideau, Passo Fundo, Brasil

- Dra. Vivien Diesel, Universidade Federal de Santa Maria, Brasil

- Dra. Vera Lucia Spacil Raddatz, Universidade Regional do Noroeste do Estado do Rio Grande do Sul, Brasil

- Dr. Waldecy Rodrigues, Universidade Federal do Tocantins, Brasil

- Dr. Walter Frantz, Universidade Regional do Noroeste do Estado do Rio Grande do Sul, Brasil

- Dr. Walter Guedes Silva, Universidade Estadual de Mato Grosso do Sul, Brasil

- Dr. Walter Marcos Knaesel Birkner, Universidade do Contestado, SC, Brasil

- Dr. Wendel Alex Castro Silva, Faculdade Novos Horizontes, Belo Horizonte, Brasil

- Dr. Wenner Glaucio Lopes Lucena, Universidade Federal da Paraíba, Brasil

- Dr. Wesley Mendes-da-Silva, Fundação Getúlio Vargas, Brasil 